\title{
„Timing“ der operativen Versorgung von Sportverletzungen am Knie
}

\author{
Ernst-Otto Münch
}

\section{Zusammenfassung}

In der Versorgung von Kniegelenksverletzungen bei Sportlern beeinflussen einige Faktoren Timing und Art der operativen Therapie. Ziel ist es, den Sportler so schnell und optimal wie möglich zurück in den Sport zu bekommen. Sofern aus logistischen Aspekten möglich, wird eine möglichst zeitnahe, wenn nicht sogar unmittelbare Versorgung angestrebt. Ist dies nicht möglich, wird mithilfe von Physiotherapie ein reizfreier Zustand des Gelenks angestrebt, um das postoperative Arthrofibroserisiko zu minimieren. Begleitend hierzu gehören auch optimale chirurgische Rahmenbedingungen zur bestmöglichen Versorgung. Auch neuere Verfahren, wie den Erhaltungsversuch von möglichst viel eigenem Gewebe wie bei der vorderen Kreuzbandrekonstruktion, sollten in Erwägung gezogen werden.

\section{Timing for the Surgical Treatment of Sporting Injuries to the Knee}

Several factors may influence the timing and procedure of surgical treatment for sporting injuries to the knee. The principle is to allow the athlete to return to sport as soon and as optimally as possible. If logistically possible, prompt or immediate surgical treatment should be the goal. If this is not possible, physiotherapy is used to eliminate inflammation in the joint and thus to prevent postoperative arthrofibrosis. This should be accompanied by the optimal conditions for surgery. Furthermore, new techniques should be considered, such as maintaining as much native tissue as possible in anterior cruciate ligament reconstruction.
Verletzungen des Kniegelenks gehören zu den häufigsten Verletzungen im Sport. Insbesondere im alpinen Skisport und bei Mannschafts- und Kontaktsportarten wie Fußball, Basketball und Handball kommt es zu teilweise schwerwiegenden Traumen, die meist operativ versorgt werden müssen. Dies trifft vor allem für Verletzungen der Kreuzbänder und Menisken zu. Es können letztendlich aber alle anatomischen Strukturen des Kniegelenks isoliert oder kombiniert betroffen sein.

Für den Operateur bedeutet die Versorgung von Sportlern immer eine besondere Herausforderung, da neben den rein medizinischen Gesichtspunkten im-

OP-JOURNAL 2016; 32: 24-26

(c) Georg Thieme Verlag KG Stuttgart · New York DOI http://dx.doi.org/10.1055/s-0042-106760 mer noch weitere Aspekte hinzukommen.

Die Aufgabe wird dabei für den Arzt umso schwieriger, je höher das Leistungsniveau des Sportlers und sein Bekanntheitsgrad (regional, national, international) sind.

Nur wenige Verletzungen im Bereich des Kniegelenks haben einen echten Notfallcharakter und erfordern ein sofortiges Eingreifen in der nächstgelegenen operativen Einrichtung, die zu einer entsprechenden Versorgung in der Lage ist. Dazu zählen bspw. Knieluxationen mit Gefäßbeteiligung oder Frakturen mit Kompartmentsyndrom. So erlitt ein alpiner Skirennläufer der Deutschen Nationalmannschaft bei einem Weltcuprennen in Norwegen eine Tibiakopf- und Unterschenkelfraktur mit Entwicklung eines Kompartmentsyndroms (Abb. 1 und 2). Ein Rücktransport nach Deutschland in eine Unfallklinik seiner Wahl war aus Zeitgründen und bei der Dringlichkeit einer operativen Behandlung nicht möglich. Er musste daher in der schnellstmöglich erreichbaren Klinik im Ausland erstversorgt werden.

Für die allermeisten Verletzungen im Sport wie Patellaluxationen, Kreuzbandrupturen, Meniskusläsionen, Knorpelverletzungen, Tibiakopffrakturen oder Sehnenrupturen gibt es jedoch ein gewisses Zeitfenster, in dem eine operative Behandlung sinnvoll und möglich ist. Allerdings kommen dann häufig außer der rein medizinischen Indikation noch andere Aspekte hinzu, die durchaus einen Einfluss auf den Operationszeitpunkt haben können.

Sportler haben aufgrund ihrer hohen Anspruchs- und Erwartungshaltung immer den verständlichen Wunsch nach einer optimalen operativen Behandlung. Dazu gehört neben der möglichst vollständigen Wiederherstellung der verletzten Strukturen auch der Wunsch nach einer möglichst schnellen Rückkehr in den Leistungssport. Um den Zeitverlust so gering wie möglich zu halten, besteht oft die Forderung nach einer sofortigen operativen Versorgung. Dieser Wunsch kommt aber oft nicht nur vom Sportler selbst, sondern auch von seinem gesamten Umfeld. Dazu gehören neben der Familie vor allem auch Trainer, Physiotherapeuten, der Verband oder Verein mit seinen Sportfunktionären und auch die gesamte Medienlandschaft und Öffentlichkeit. So kann es durchaus sein, dass z. B. ein hochbezahlter Fußballprofi auch mitten in der Nacht und ohne medizinische Dringlichkeit wegen einer Meniskusläsion (Abb. 3) operiert wird nur um dem Druck einer möglichst schnellen Rückkehr in den Leistungssport nachzugeben. 


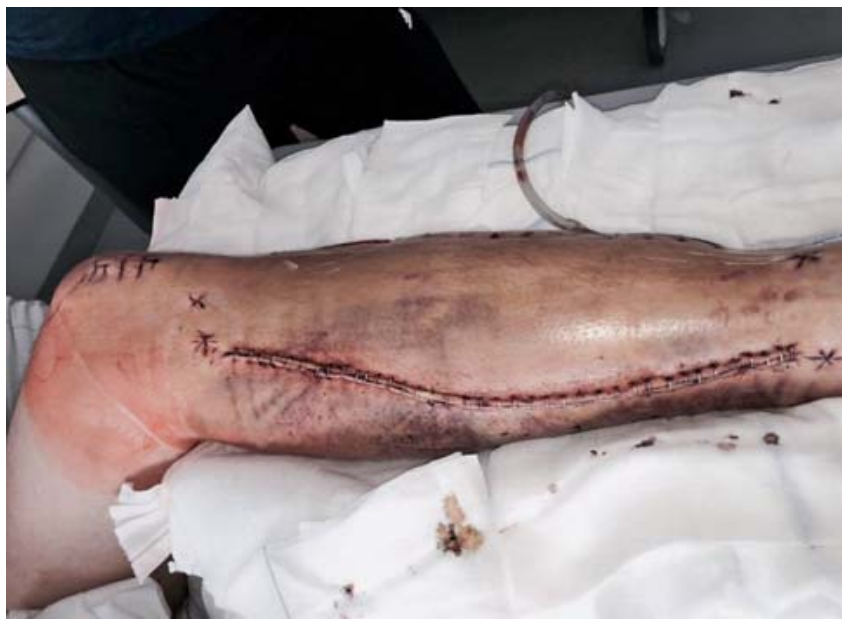

Abb. 1 Kompartmentsyndrom nach Fraktur.

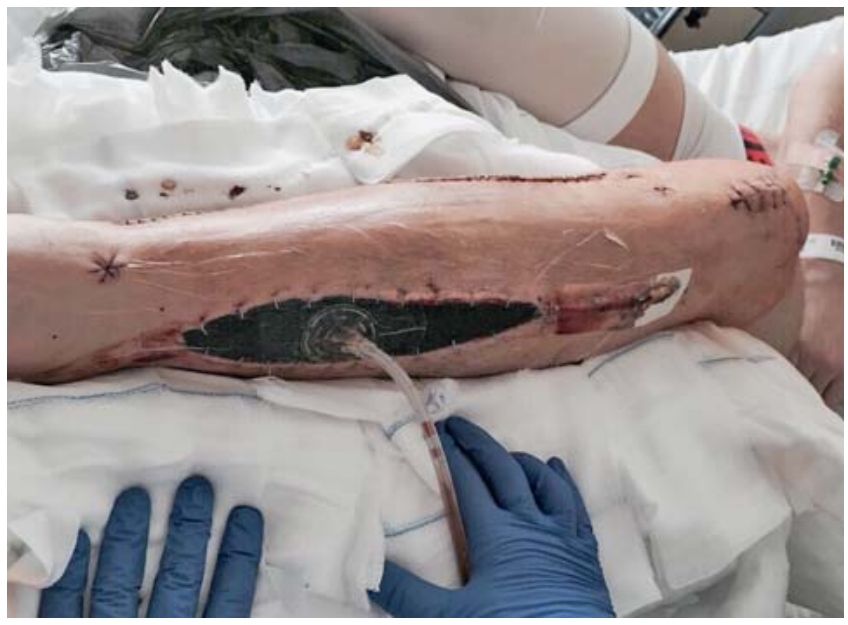

Abb. 2 Kompartmentsyndrom bei einem Sportler.
Generell wird beim Leistungssportler im Falle einer Knieverletzung mit einer OPIndikation aber schon eine frühzeitige Operation angestrebt.

Der wesentliche Grund dafür ist, den Sportler so bald wie möglich wieder in Training und Wettkampf zurückzuführen. Die Operation sollte dann nicht nur möglichst schnell, sondern am besten auch unter optimalen Voraussetzungen durchgeführt werden. Dazu gehören neben einem großen Erfahrungsgrad des Operateurs auch ein erfahrener Anästhesist, ein routiniertes OP-Team und beste räumliche, apparative und instrumentelle Voraussetzungen.

Auch vonseiten des Sportlers gibt es Umstände, die auf den bestmöglichen Operationszeitpunkt Einfluss haben können. So sollte der Patient wegen der Anästhesie nüchtern sein. Die lokalen Weichteilverhältnisse (Hautläsionen, Schwellungen, Verschmutzungen etc.) müssen berücksichtigt werden. Auch die psychische Verfassung des Athleten darf nicht außer Acht gelassen werden. Nicht um jeden Preis darf daher eine Operation so schnell wie nur irgend möglich erfolgen. Insgesamt besteht aber durchaus Einigkeit über eine frühzeitige operative Behandlung bei Sportverletzungen mit OPIndikation.

Kontrovers wird lediglich das Vorgehen bei akuten Kreuzbandrupturen diskutiert.

So wurde über lange Zeit die Auffassung vertreten, dass vordere Kreuzbandrisse erst nach einem Intervall von 6 Wochen operiert werden dürften. Der Grund hierfür war ein erhöhtes Risiko für eine Arthrofibrose, wenn in der unmittelbar posttraumatischen Phase operiert wurde. Bis zur Operation hat man versucht, mit Krankengymnastik, Physiotherapie und medikamentöser Behandlung den Schwellungszustand des Kniegelenks zu reduzieren und die Beweglichkeit $\mathrm{zu}$ verbessern. Nicht selten wurde auch primär eine Arthroskopie nach der Verletzung durchgeführt mit Ausspülung des Hämarthros und teilweise auch Resektion der Kreuzbandstümpfe und Versorgung von Begleitverletzungen, z.B. an Knorpel und Menisken. Die eigentliche vordere Kreuzbandoperation wurde dann meist erst 6 Wochen später durchgeführt.

Dieses Vorgehen praktizieren wir heute jedoch nur noch selten. Aufgrund einer eigenen Untersuchung in unserer Klinik an fast 400 Patienten, bei denen wegen einer postoperativen Arthrofibrose nach vorderer Kreuzbandplastik eine Arthrolyse durchgeführt werden musste, konnten wir feststellen, dass das Zeitintervall zwischen Verletzung und Operation nicht der entscheidende Faktor war. Vielmehr war der klinische Reizzustand des Gelenks und die subjektive Schmerzhaftigkeit bedeutsam. Es konnte ein signifikanter Zusammenhang zwischen einem präoperativen Reizzustand (Schwellung, Überwärmung, Schmerzen) mit Bewegungseinschränkung und einer postoperativen Arthrofibrose identifiziert werden. Der OP-Zeitpunkt darf daher nicht als isolierter Risikofaktor gesehen werden.

Bei den heutigen Sportveranstaltungen ist eine sofortige ärztliche Versorgung in aller Regel vorhanden und gut organisiert. Auch die bildgebende Diagnostik kann für den Leistungssportler meist in-

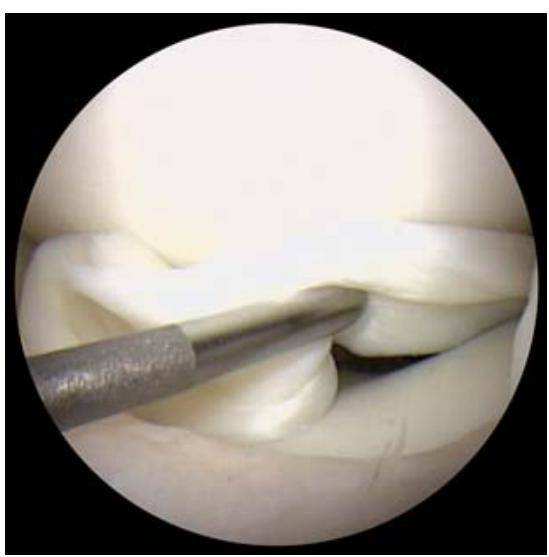

Abb. 3 Eingeklemmter Meniskus.

nerhalb von Stunden ermöglicht werden. Dadurch können die Athleten in vielen Fällen so frühzeitig operiert werden, dass der schmerzhafte und prognostisch ungünstige Reizzustand noch gar nicht eingetreten ist. Der Vorteil für den Leistungssportler besteht v.a. in einem Zeitgewinn für eine schnellere Rückkehr in Training und Wettkampf.

Die Möglichkeiten einer vorderen Kreuzbandrekonstruktion sind inzwischen vielfältig. In aller Regel wird das vordere Kreuzband durch autologes Sehnengewebe ersetzt. Hierfür gibt es verschiedene Optionen (Beugesehnen, Patellarsehne, Quadrizepssehne). Auch die Operationstechniken sind variabel. Angestrebt wird allerdings immer eine möglichst anatomische Rekonstruktion.

Kreuzbandrupturen können ein sehr unterschiedliches Erscheinungsbild haben. Nicht immer sind Kreuzbandrisse komplett. Es kommt durchaus vor, dass sogar einzelne Bündel des vorderen Kreuzbands in der Kontinuität erhalten blei- 


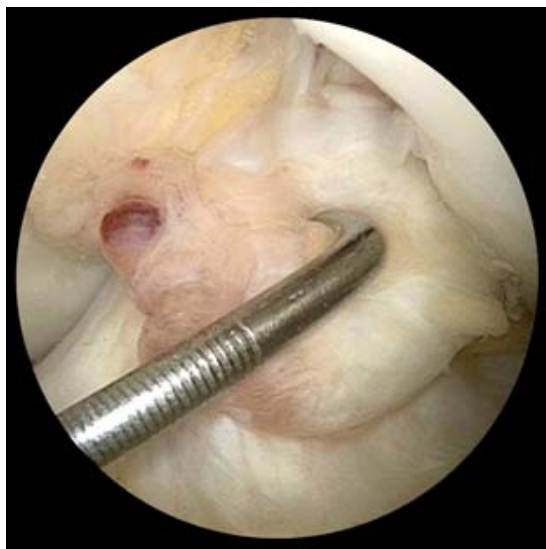

Abb. 4 Proximale vordere Kreuzbandruptur.

ben. Bei Rupturen im proximalen Drittel verbleiben oft kräftige und lange tibiale Reste.

Ein neuer Trend besteht nun darin, möglichst viel vom verletzten vorderen Kreuzband zu erhalten.

Dazu wird der tibiale vordere Kreuzbandrest durch eine Naht gefasst und refixiert. (Abb.4, 5 und 6) Zusätzlich erfolgt eine Augmentation durch eine Beugesehne oder auch alloplastisches Material. Man erwartet dadurch, wichtige Nervenendigungen zu schonen, die für die sensomotorischen Fähigkeiten bedeutsam sind. Davon wiederum erhofft man sich Vorteile für die Rehabilitation und eine schnellere und bessere Rückkehr in den Sport.

Allerdings kommen solche Verfahren mit Erhalt von natürlichen Kreuzbandanteilen nur infrage, wenn der Eingriff innerhalb der ersten 3 Wochen nach Trauma durchgeführt werden kann. Dabei ist es aber wiederum wichtig, dass kein schmerzhafter Reizzustand des Kniegelenks vorliegt.

Nicht immer lässt sich jedoch die von uns favorisierte Form der Frühversorgung realisieren. So hatten wir im Deut-

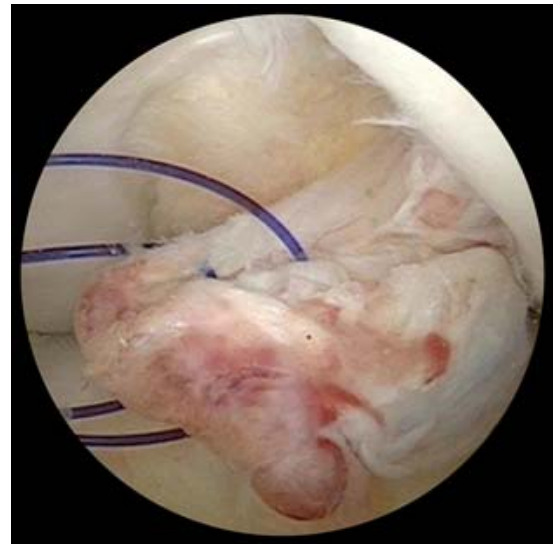

Abb. 5 Fassen des vorderen Kreuzbandstumpfs durch Naht.

schen Skiverband schon mehrfach die Situation, dass sich ein alpiner Rennläufer in USA, Kanada oder Südamerika verletzt hat und sich der Rücktransport aufgrund der großen Entfernung und teilweise schwierigen Flugverbindung sehr verzögert hat. Nicht selten vergingen zwischen Verletzung und Eintreffen bei uns mehr als 40 Stunden. Die Kniegelenke waren durch das Trauma und den langen Transport oft so massiv geschwollen, schmerzhaft und in der Beweglichkeit eingeschränkt, dass eine operative Frühversorgung kontraindiziert war. In diesen Fällen wurde eine präoperative Vorbehandlung mit Physiotherapie und Krankengymnastik durchgeführt, bis der klinische Untersuchungsbefund und die Schmerzhaftigkeit eine risikoarme Operation zuließen. Dies war meist zwischen 3 und 6 Wochen nach der Verletzung möglich.

Gelegentlich wurde auch ein zweizeitiges Verfahren praktiziert. Dabei wurde jeweils ein dislozierter und eingeklemmter Meniskus primär reponiert und refixiert. Die Kreuzbandrekonstruktion erfolgte dann mehrere Wochen später.

Zusammenfassend lässt sich zum Operationszeitpunkt einer Knieverletzung beim Sportler Folgendes sagen:

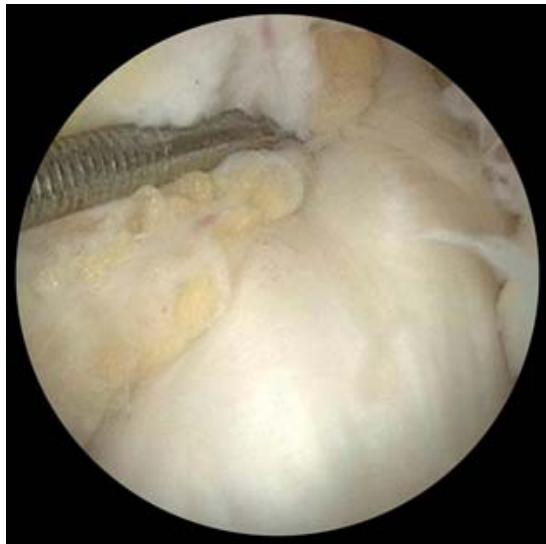

Abb. 6 Vorderes Kreuzband nach Refixation durch Naht und Augmentation.

- absolute Notfälle mit einer sofortigen OP-Notwendigkeit sind sehr selten (Luxationen mit Nerven- und Gefäßläsion, Kompartmentsyndrom),

- die allermeisten Knieverletzungen sollten schnell, aber unter optimalen Voraussetzungen durchgeführt werden,

- bei akuten Reizzuständen eines traumatisierten Kniegelenks (massive Schwellung, Schmerzen, Bewegungseinschränkung) ist ein verzögertes oder auch zweizeitiges Vorgehen indiziert,

- operative Versorgungen von vorderen Kreuzbandrupturen werden differenziert, individuell und in unterschiedlichen Zeitintervallen durchgeführt.

Literatur beim Verfasser.

\section{Dr. Ernst-Otto Münch}

Orthopäde, Sportmedizin, Diplomsportlehrer Leitender Mannschaftsarzt der alpinen Ski-Nationalmannschaft

OCM (Orthopädische Chirurgie München)

Steinerstraße 6

81369 München ernst-otto.muench@ ocm-muenchen.de 